\title{
YOGA FOR PALLIATIVE CARE NURSES
}

\author{
Renuka K, Dean, Nursing Faculty, \\ Principal of KGNC,
}

Anbu $M$, Lecturer, Dept. of Medical Surgical Nursing.

Kasturba Gandhi Nursing College - Mahatma Gandhi Medical College and Research Institute Campus Pillaiyarkuppam, Puducherry - 607403, India

?

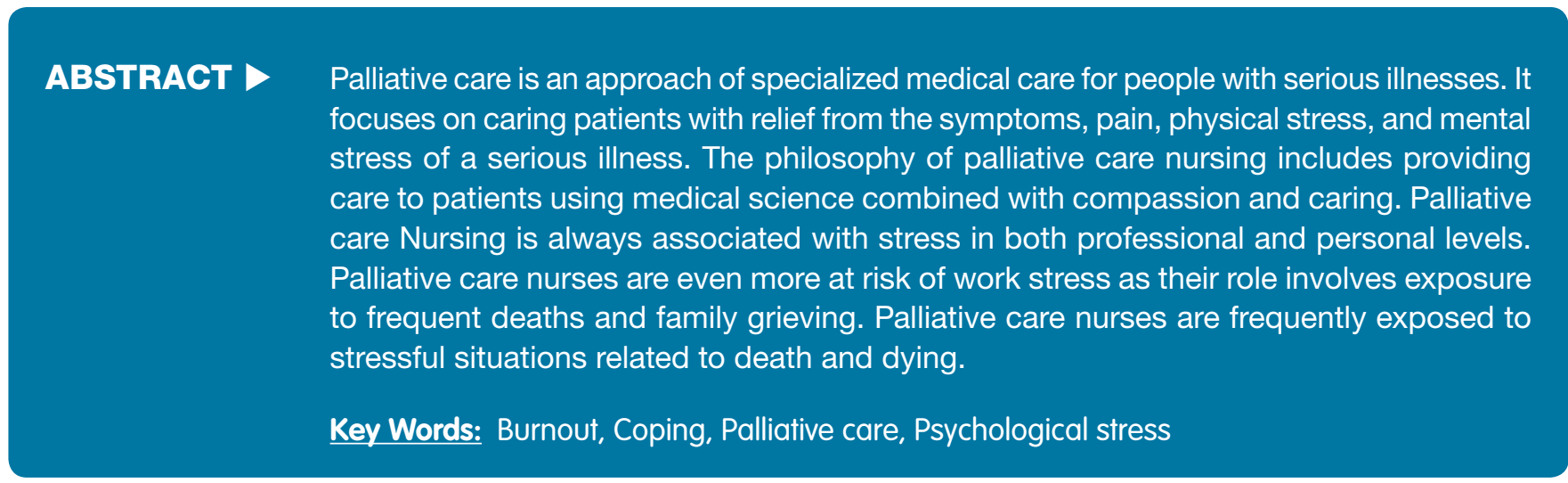

\section{INTRODUCTION}

Palliative care is unique in its holistic approach to care. It involves treating not only pain and physical symptoms but also the psychological, social, and spiritual needs of patients. Given the characteristics of their profession, nursing staff are more heavily involved in the direct care of these patients, both in residential and hospital settings. ${ }^{1-3}$ When a patient dies in a hospital or residential center, the emotional burden that comes with exposure to death that had previously fallen primarily on families is now also assumed by health care staff. ${ }^{4}$ When one is exposed to such events repeatedly, the phenomenon generates stress, anxiety, depression, and emotional fatigue among nursing professionals and changes in personality variables. ${ }^{5-6}$. These stimuli may also trigger the burnout syndrome, which is defined as "a syndrome of emotional exhaustion, depersonalization, and reduced personal achievement that can develop in individuals who carry out activities oriented toward working with other people."7 Burnout syndrome is associated with, increased risk of employee absenteeism and increased intention to quit their employment the syndrome results in reduced efficacy and efficiency among nursing staff in work-related settings. ${ }^{8,9}$ This can affect workplace environment by increasing levels of dissatisfaction and rates of absenteeism and low productivity, conflict between co-workers, and higher turnover rates. ${ }^{10}$

Wilkes and Beale ${ }^{13}$ conducted a qualitative study on Exploration of perceived stressors for urban and rural palliative care nurses working Australia. The study concluded that major stressors for both group of nurses were impact of family relationships and role conflict in the community. Furthermore the study also identified 24-hour service over great distances and lack of financial resources for rural nurses, were the additional stressors and urban nurses reported stressful situations arising when families had coping problems or personality issues, families with whom communication was 
difficult, families who were physically and emotionally distressed in caring for a family member at home, and where identification with the family or the patient was difficult ${ }^{13}$

Grau-Arberola et al $^{14}$ conducted a study to determine burnout levels among palliative nursing staff of a particular hospital at a particular time and conducted the same test 1 year later. The study revealed that a significant increase in emotional fatigue levels and depersonalization had occurred over time among the palliative care nursing staff. Working experience, communication process and environmental factors are contributing to the stress experienced by the palliative care nurses ${ }^{14}$

As palliative care nurses, are very good at taking care of others, often they fall short of taking care of themselves. Physical activity is a huge piece of self-care, not just for its physical benefits but for emotional and mental benefits, as well. ${ }^{11}$ The aim of this article is not removing the stress completely, because stress is part of the life, but response to stress can be in an unhealthy way, and the role of the nurse at this time is helping herself in health promotion strategies for reduction and management of the stress. These strategies include physiologic, behavioral, and experimental responses, which can result in a different outcome in physical and mental health ${ }^{15}$. Stress coping strategies are the efforts that individuals make to enhance their adaptation with the environment, and to prevent negative outcomes of overload. ${ }^{12}$ Various techniques are employed to control or lower stress, and to amend stress coping strategies and behavior ${ }^{16}$. It is imperative for the palliative care nurses to manage the stress level for themselves to promote good professional and personal development. The practice of Yoga has proved to revert back the nurses to a state of healthy state of body and mind and to prevent stress disorders ${ }^{17}$.

Yoga, has been studied and emphasized by scientists as a technique to relax the mental state and control stress through internal and external sources. These techniques are used as both preventive and recovery methods. ${ }^{18}$ Yoga is the most ancient action-discipline system known in the world whose exercises help people to cope with stress.

\section{YOGA - A STRESS BUSTER FOR PALLIATIVE CARE NURSES.}

Yoga, developed thousands of years ago, is recognized as a form of mind-body exercises improve muscle strength, flexibility, blood circulation and oxygen uptake as well as hormone function. In addition, the relaxation induced by meditation helps to stabilize the autonomic nervous system with a tendency towards parasympathetic dominance.

In a study in 2007 to investigate the effect of Tai Chi Yoga and meditation and promotion of nurses' health and their problem-solving ability, it was revealed that Yoga promoted nurses' health, their decisionmaking ability, and their concentration on giving care in critical conditions. ${ }^{19}$ Another study conducted on 17 nonprofessional caretakers showed that there was a noticeable increase in their stress coping strategies after 8 weeks of Yogic exercises. ${ }^{20}$ Since the main goal of nursing is to promote the level of public health, and as inappropriate responses and application of nonefficient coping strategies can jeopardize individuals' health, taking actions toward promotion of stress coping strategies and reduction of stress side effects are considered among nurses' duties. ${ }^{21}$

Yoga and meditation are part of a holistic approach to self-healing that brings about harmony between body, mind and soul and helps in relieving numerous aliments.

- Brings about harmony between body, mind and soul

- Promotes the process of self-healing

- Controls blood pressure

- Slows down the process of aging

- Relieves stress and anxiety

- Improves functioning of the nervous system

- Increases concentration and focus

- Weight gain

- Improves flexibility and posture

- Boosts energy

\section{YOGA STEPS FOR PALLIATIVE CARE NURSES}

Instead of reaching out for antidepressant pills, bring out yoga mat and try these poses when you feel low and depressed and see your mood perk up instantly.

\section{Child Pose (Balasana)}

The child pose or balasana is undoubtedly the most relaxing yoga pose that helps in calming the mind and 


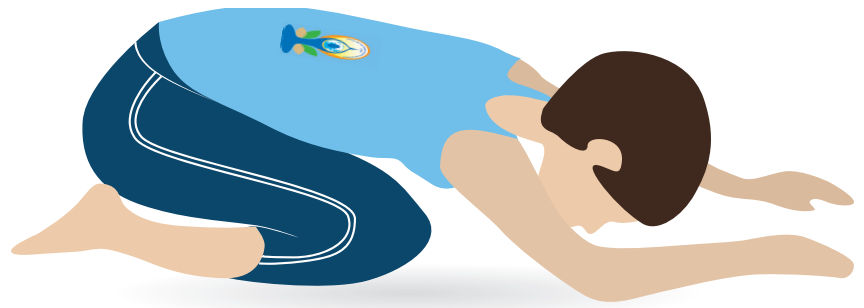

rejuvenating the body with energy. It is also an effective yoga pose for relieving lower back pain, hip strain and shoulder and neck stiffness. It has a healing and restorative power that helps in providing mental and emotional relief. Sit on the yoga mat kneeling while bringing your knees together and resting your buttocks on your feet. Now, slowly lower your torso over your thighs exhaling so that your forehead touches the mat and your hand rest on the floor. Hold the position for 30 seconds to 1 minutes breathing regularly and come back to the starting position. This makes one repetition. Repeat it 3 to 4 times. 22

\section{Standing Forward Bend (Uttanasana)}

The standing forward bend is the ideal yoga pose to reduce stress. It helps in preserving proper functioning of the nervous system by improving blood supply throughout the body. It also helps in stretching and relaxing the spine and increasing flexibility. It tones the abdominal muscles and organs, and is the perfect yoga pose for weight loss. Stand with your feet closed and arms resting on the side. Raise your hand above your head while inhaling and bend your torso forward while exhaling. Touch the ground with both your hands, keeping your legs straight. Hold the pose for 30 seconds with normal breathing rhythm. Come back to the starting position. This makes 1 repetition. Repeat it 5 to 10 times $^{22}$

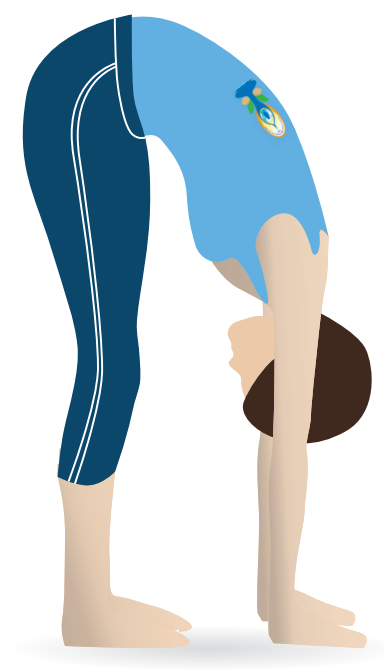

\section{Down Dog (Adho Mukha Savasana)}

The down dog or the downward facing dog pose is an amazing stress-relieving yoga pose that helps in waking up the senses and reduces fatigue and tiredness. It helps

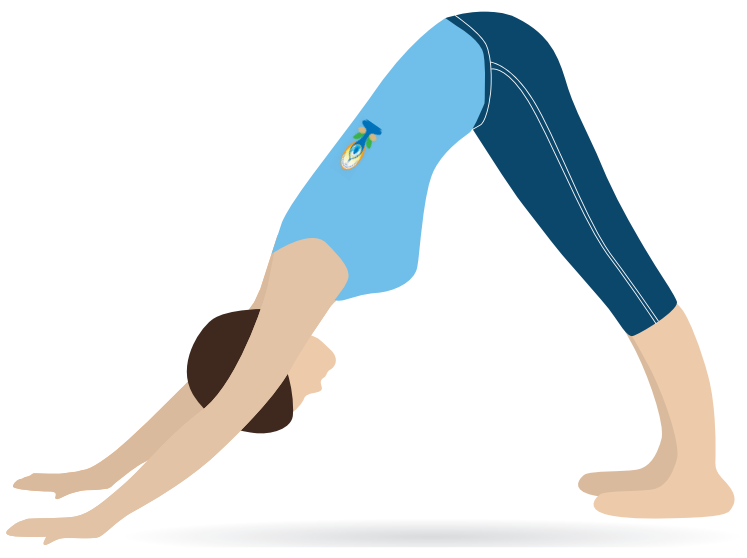

in improving bone density and preventing bone diseases such as osteoporosis in women. It rejuvenates the body by improving blood circulation, and this yoga pose can also help in relieving neck pain by reducing stiffness of the upper body. Get down on your knees and hands so that your back remains straight and parallel with the floor. Now, push up your hips by straightening the knees and elbows so that your body forms an inverted $\mathrm{V}$ shape, Hold the pose for 30 seconds to 1 minute while breathing normally. Return to the starting position exhaling slowly. This makes 1 repetition. Repeat it 5 to 6 times. ${ }^{22}$

\section{Bridge Pose (Setu Bandha Sarvangasana)}

The bridge pose (Setu Bandha Sarvangasana) is the best yoga pose for stress and anxiety. It helps in stretching the muscles of the back and the leg and is an effective exercise for toning the thighs and hips. In addition, it also helps in controlling high blood pressure and reduces backaches, headaches, sleep disorders and fatigue. Lie flat on the yoga mat and bend your knees to rest your feet close to the buttocks. Your feet should be placed

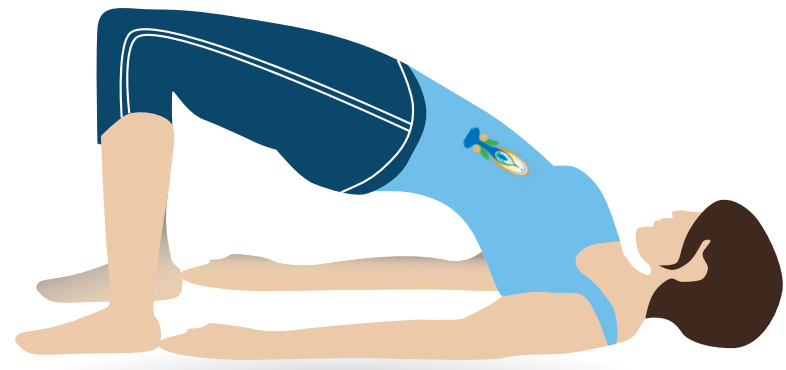


hip width apart. Now, lift your hips upward by pressing the arms on the floor and lift your breastbone as well while keeping your head fixed facing to the ceiling. Remain in the position for 30 seconds to 1 minute and come back to the starting position. This makes 1 repetition. Repeat it 5 to 6 times. $^{22}$

\section{Extended Triangle Pose (Utthita Trikonasana)}

The extended triangle pose is the ideal yoga pose for stress management that helps in full-body stretching. It helps in increasing flexibility, improving digestion and relieves the problems of anxiety, depression and fatigue. It addition, it also increases bone density and reduces the risk of developing osteoporosis. It also helps in toning the oblique muscles and is effective to reduce fat. Stand straight with your feet approximately 4 feet apart, raise your arms on both sides and keep them parallel to the floor,. Now turn your right foot out by 90 degrees and bend your torso to the right side while

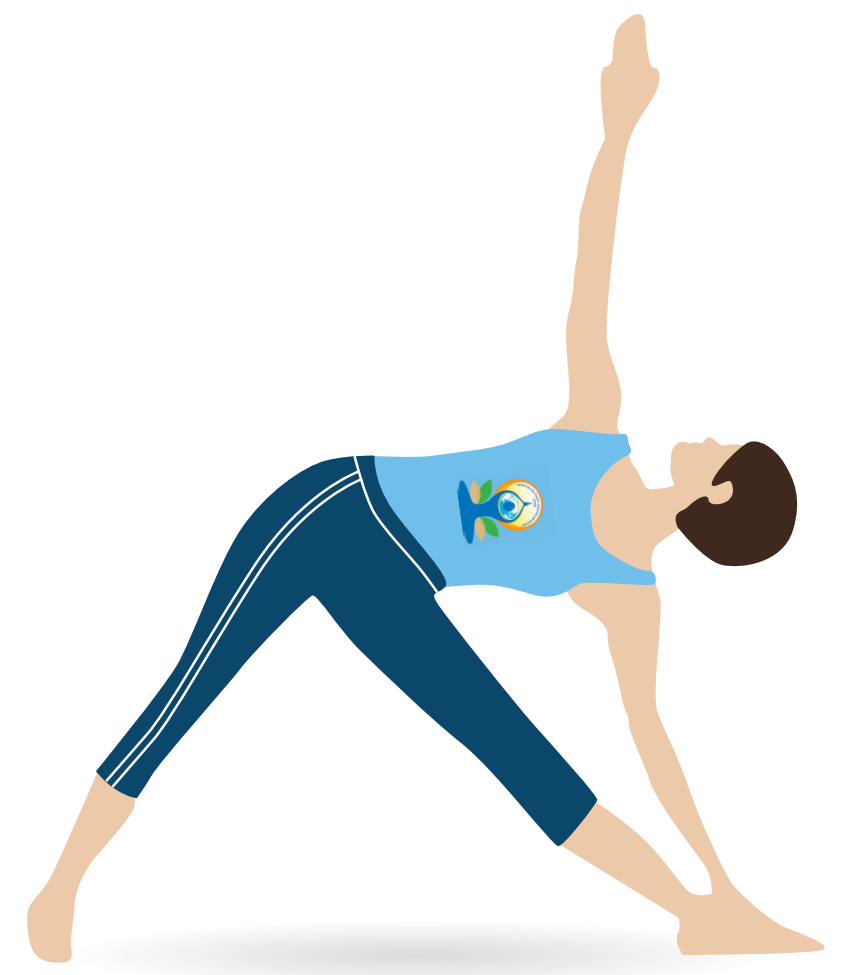

exhaling, and hold your right calf with your right hand while pointing your face and left hand towards the ceiling. Hold the position for 30 seconds and come back to the starting position while inhaling. This is 1 repetition. Now repeat the entire movement on the left side. Repeat it 5 to 6 times on both sides. ${ }^{22}$

\section{Cat Pose (Marjaryasana)}

The cat pose is the best pose for stress relief that helps in stretching the spine and toning the muscles and organs of the abdomen. It helps in improving overall health by relieving digestive problems. Get on all fours with your wrists directly under your shoulders and your knees directly under your hips. Now, exhale and pull your spine inward to form a curve pointing towards the

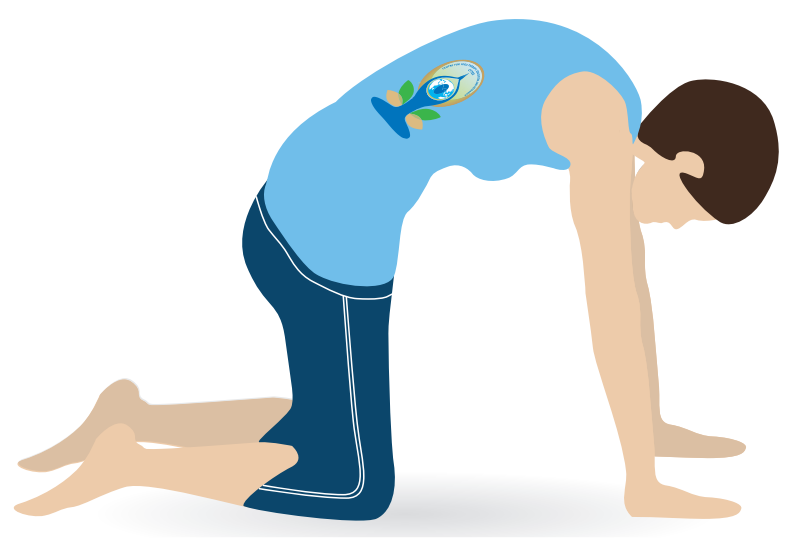

ceiling. Bend your head toward the floor, but make sure your chin should not touch the breast. Hold the pose for 30 seconds and come back to the starting position. In most cases, the cat pose is combined with cow pose which you can do by inhaling and dropping your belly towards the mat while pointing your head upward. These 2 poses are to be repeated 5 to 6 times holding each pose for 30 seconds alternatively ${ }^{22}$

\section{Extended Puppy Pose (Uttana Shishosana)}

The extended puppy pose is one of the most simple yoga exercises that helps in relaxing the body by stretching the spine and shoulder, thus reducing stiffness. It is useful for correcting body posture, increasing flexibility and rejuvenating the mind and the body. This pose helps in toning the hips and back and is one of the best yoga poses to lose weight after pregnancy. Begin on all fours and slowly lower your chest towards the floor by sliding your arms forward but make sure that your elbows don't touch the ground. Drop your head so that

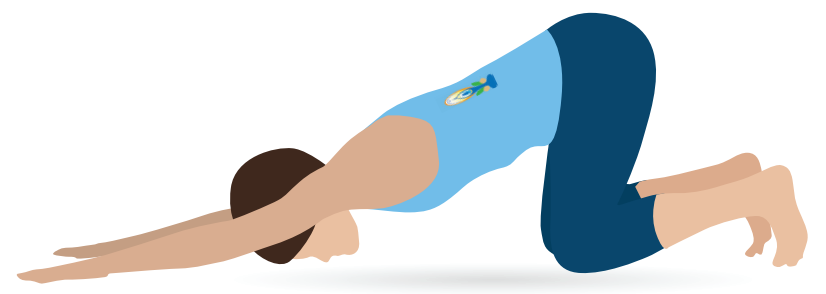


it touches the floor. Keep your neck relaxed and hold the pose for 30 seconds to 1 minute while breathing normally. Now, come back to the starting position while exhaling. This makes one repetition. Repeat it 5 to 6 times. $^{22}$

\section{Corpse Pose (Savasana)}

Last but not the least comes the corpse pose or Savasana, the ultimate yoga pose for stress relief. No yoga session is complete without the corpse pose which is the most calming and relaxing of all poses. It transports the body to a state of deep rest and relaxation. This yoga pose helps in promoting sleep, lowering high blood pressure and calming the nervous system. Lie down on

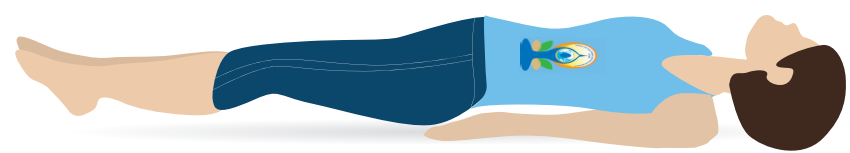

the yoga mat with any cushion or pillow for support. Keep your feet slightly apart and relax your knees and toes. Place your arms on the side of your body, your palms open and facing the ceiling. Close your eyes and breathe slowly and deeply to relax your body and mind. Stay in this posture for 10 minutes and end your yoga session in a relaxed and rejuvenated way. ${ }^{22}$

\section{CONCLUSION}

This article identifies the value of support of yoga to improve palliative care nurses' self-care and overcome burnout. Palliative care nurses experience work-related stress which emphasizes the need for weekly regular yoga practice. It would significantly decrease workrelated stress and increases the stress adaptation of palliative care nursing professionals which will help them to give a quality care.

\section{REFERENCES}

1. Higginson IJ, Finlay IG, Goodwin DM, Hood K, Edwards AG, Cook A, Douglas HR, Normand CE. Is there evidence that palliative care teams alter end-of-life experiences of patients and their caregivers? Journal of pain and symptom management 2003; 25 (2): 150-168.

2. Lim J, Bogassian F, Ahern K. Stress and coping in Australian nurses: a systematic review. Int Nurs Rev 2010; 57(1): $22-31$

3. Donovan RO, Doody 0, Lyons R. The effect of stress on health and its implications for nursing. Br J Nurs 2013; 22: 969-70.

4. John OP, Donahue EM, Kentle RL. The Big Five Inventory. Berkeley, CA: University of California (Institute of Personality and Social Research); 1991.

5. Borritz M, Rugulies R, Bjorner JB, Villadsen E, Mikkelsen OA, Kirstensen TS. Burnout among employees in human service work: design and baseline findings of the PUMA study. Scandinavian Journal of Public Health 2006; 3481:49-58.

6. Edwards D, Burnard P, Owen M, Hannigan B, Fothergill A, Coyle D. A systematic review of the effectiveness of stress management interventions for mental health professionals. J Psychiatr Ment Health Nurs 2003;10:370-71.

7. Warshawsky NE, Havens DS. Nurse manager job satisfaction and intent to leave. Nurs Econ 2014; 32: 32-39.

8. Westermann C, Kozak A, Harling M, Nienhaus A. Burnout intervention studies for inpatient elderly care nursing staff: systematic literature review. Int J Nurs Stud 2014; 51:63-71.

9. Garrosa E, Moreno-Jime'nez B, Liang Y, Gonza'lez JL. The relationship between socio-demographic variables, job stressors, burnout, and hardy personality in nurses: an exploratory study. Int J Nurs Stud 2008;45:418-27

10. Go'mez C, GandoyM, PugaA, ClementeM, Mayán JM. Factores depersonalidad del personal de enfermería de unidades de cuidados paliativos. (Personality traits of palliative care unit nursing staff). Gerokomos 2012;23:110-13.

11. Vachon ML. Caring for the caregiver in oncology and palliative care. Semin Oncol Nurs 1998;14(2): 152-57

12. Wakefield A Nurses' responses to death and dying: a need for relentless self-care. Int J Palliat Nurs :2000, 6(5): 245-51

13. Wilkes LM, Beale B : Palliative care at home: stress for nurses in urban and rural New South Wales, Australia. Int J Nurs Pract $2001 ; 7(5)$ : 306-13

14. Grau-Alberola E,Gil-Monte PR,García-Juesas JA, Figueirido-FerrazH.Incidence of burnout among Spanish nursing professionals: a longitudinal study. Int J Nurs Stud. 2010;47:1013-20

15. Weinstein N, Brown KW, Ryan R. A multi-method examination of the effects of mindfulness on stress attribution, coping, and emotional well-being. J Res Pers 2009; 43:374-85.

16. Forozandeh N, Delaram M. Effect of cognitive behavioral therapy on coping methods non-medical student university of medical sciences. Shahre Kord Univ Med Sci J 2003; 5: 26-34.

17. Akochekian SH, Rohafza HR, Hasanzadeh A, Mohammad Shrifi H. Associated with social support and coping strategies in a psychiatric ward nurses. J Med Sci Gilan 2008; 18: 41-6.

18. Rizzolo D, Zipp GV, Stiskal D, Simpkins S. Stress management strategies for students: The immediate effects of yoga, humor, and reading on stress. J Coll Teach Learn 2009; 6: 79-88.

19. Raingruber B, Robinson C. The effectiveness of Tai Chi, yoga, meditation, and Reiki healing sessions in promoting health and enhancing problem solving abilities of registered nurses. Issues Ment Health Nurs 2007; 28:1141-55

20. Van Puymbroeck M, Payne L, Hsieh PC. A phase I feasibility study of yoga on the physical health and coping of informal caregivers. Evid Based Complement Alternat Med 2007;75:11-17.

21. Ramezani S. Effect of humor education on mental health and coping styles of nurses in emergency departments in hospitals affiliated to medical sciences university of shiraz, MS Thesis. Shiraz, Iran: Shiraz University of Medical Sciences; 2006.

22. Yoga Journals for Healthy Minds and Healthy Body. Cruz bay publishers:2014 